\title{
Influence of the Saturation Ratio on Concrete Behavior under Triaxial Compressive Loading
}

\author{
Xuan-Dung Vu, ${ }^{1,2}$ Matthieu Briffaut, ${ }^{1,2}$ Yann Malecot, ${ }^{1,2}$ \\ Laurent Daudeville, ${ }^{1,2}$ and Bertrand Ciree ${ }^{3}$ \\ ${ }^{1}$ Université Grenoble Alpes, 3SR, 38000 Grenoble, France \\ ${ }^{2}$ CNRS, 3SR, 38000 Grenoble, France \\ ${ }^{3}$ Institut de Radioprotection et de Sûreté Nucléaire, DSR/SAMS/BAGCS, 92260 Fontenay-aux-Roses, France
}

Correspondence should be addressed to Yann Malecot; yann.malecot@ujf-grenoble.fr

Received 31 October 2014; Accepted 16 March 2015

Academic Editor: Arkady Serikov

Copyright (C) 2015 Xuan-Dung Vu et al. This is an open access article distributed under the Creative Commons Attribution License, which permits unrestricted use, distribution, and reproduction in any medium, provided the original work is properly cited.

\begin{abstract}
When a concrete structure is subjected to an impact, the material is subjected to high triaxial compressive stresses. Furthermore, the water saturation ratio in massive concrete structures may reach nearly $100 \%$ at the core, whereas the material dries quickly on the skin. The impact response of a massive concrete wall may thus depend on the state of water saturation in the material. This paper presents some triaxial tests performed at a maximum confining pressure of $600 \mathrm{MPa}$ on concrete representative of a nuclear power plant containment building. Experimental results show the concrete constitutive behavior and its dependence on the water saturation ratio. It is observed that as the degree of saturation increases, a decrease in the volumetric strains as well as in the shear strength is observed. The coupled PRM constitutive model does not accurately reproduce the response of concrete specimens observed during the test. The differences between experimental and numerical results can be explained by both the influence of the saturation state of concrete and the effect of deviatoric stresses, which are not accurately taken into account. The PRM model was modified in order to improve the numerical prediction of concrete behavior under high stresses at various saturation states.
\end{abstract}

\section{Introduction}

The upcoming need for concrete structures designed against impulsive and extreme loads due to natural hazards, industrial accidents, or terrorist attacks remains an important issue. Predicting the response of such a structure, subjected to this type of loading and characterized by a high mean stress generated in the impact zone, requires constitutive modeling capable of reproducing material behavior within this loading range (high triaxial stresses and high strain rates). Improving the knowledge on the constitutive behavior of concrete under impact is a strategic issue for many sensitive infrastructures (e.g., nuclear power plants). Protective concrete structures, like nuclear reactor containment vessels, are typically massive and remain saturated at their core several years after casting, while their surfaces dry quickly in contact with air [1].

In the vicinity of the zone submitted to a hard impact (for instance the fall of an aircraft turbine on a containment vessel) a triaxial stress state occurs characterized by a compression with lateral confinement. The behavior of wet concrete may differ substantially from that of dry concrete [2]. Consequently, quantifying the influence of water content on concrete behavior is necessary in order to analyze the vulnerability of massive concrete structures to impacts.

The high-capacity triaxial press GIGA allows testing of concrete samples under various loading paths and concrete compositions [2-9].

In this paper, triaxial tests up to $100 \mathrm{MPa}$ of confining pressure performed on concrete with different degrees of saturation will be presented and analyzed. Some results of tests carried out at very high confining pressure $(600 \mathrm{MPa})$ will be discussed so as to bring out the effect of the saturation ratio. These tests will be simulated thanks to the coupled damage plasticity model PRM [10]. The model changes necessary to improve predictions will be introduced also and the influence of these changes will be highlighted 
by comparing the two model versions with experimental results.

\section{Description of the Experimental Device and Concrete Samples}

2.1. General Description of the GIGA Press Used for Triaxial Tests. The GIGA press allows loading cylindrical concrete specimens $7 \mathrm{~cm}$ in diameter and $14 \mathrm{~cm}$ high to a confining pressure up to $0.85 \mathrm{GPa}$ and a maximal axial stress of $2.3 \mathrm{GPa}$. The large sample size (compared to the high stress level) allows testing real concrete samples with an aggregate size able to reach $8 \mathrm{~mm}$ (Figure 1).

2.2. Composition of the Concrete Mix Tested. The high performance concrete mix studied herein was designed for the concrete slabs tested during the benchmark project "Improving the Robustness of Assessment Methodologies for Structures Impacted by Missiles (IRIS)" conducted by the OECD's Nuclear Energy Agency (NEA) [11]. Concrete samples were manufactured by the Finnish laboratory VTT (Technical Research Center, Finland). The composition and properties of this VTT concrete are given in Table 1.

The concrete used in this study is representative of that selected for a nuclear power plant. Its unconfined strength is roughly $67 \mathrm{MPa}$ for simple compressive stresses and approximately 4.5 $\mathrm{MPa}$ for tensile stresses. The samples were prepared for being tested with a triaxial confining pressure varying from $0 \mathrm{MPa}$ to $600 \mathrm{MPa}$. Porosity and degree of saturation measurements were carried out prior to testing (Table 1).

The maximum aggregates size used in the concrete mix is $8 \mathrm{~mm}$. For triaxial tests, specimens $70 \mathrm{~mm}$ in diameter and $140 \mathrm{~mm}$ in height allow obtaining a Representative Elementary Volume (REV) with a minimum dimension of 3 to 5 times the largest aggregate. This specification serves to avoid significant variability in results due to the presence of large aggregates. To prevent edge effects due to sample faces, the samples were cored and rectified with water.

\section{Experimental Results of Triaxial Tests}

The concrete unconfined compressive strength increase with the loading rate observed by some authors [12] is mainly due to the lateral confinement induced by inertial forces [13] that depend on both the size of samples and the strain rates. Concrete is a cohesive-frictional material and thus the apparent strength depends on the mean stress. The scientific community now considers that this rate effect is not intrinsic to the material [12] and then should not be considered in the concrete constitutive model. Thus quasistatic experiments are sufficient to calibrate the model under triaxial compression.

This section presents triaxial compression test results for a confining pressure varying from $0 \mathrm{MPa}$ to $600 \mathrm{MPa}$.
TABLE 1: Concrete mix specifications and main properties.

\begin{tabular}{lc}
\hline Concrete mix $\left(\right.$ for $\left.1 \mathrm{~m}^{3}\right)$ & \\
Gravel $(0.5 / 8)(\mathrm{kg})$ & 925.9 \\
Sand $(\mathrm{kg})$ & 646.1 \\
Water $(\mathrm{kg})$ & 215 \\
Cement $(\mathrm{CEM}$ II B 42.5$)(\mathrm{kg})$ & 489 \\
Fly ash $(\mathrm{kg})$ & 88 \\
Superplasticizer $(\mathrm{kg})$ & 6.33 \\
Density $\left(\mathrm{kg} / \mathrm{m}^{3}\right)$ & 2370 \\
Main concrete properties & \\
Compressive strength $(\mathrm{MPa})$ & 67 \\
Porosity accessible to water $(\%)$ & 12 \\
Cement paste volume $\left(\mathrm{m}^{3}\right.$ for $1 \mathrm{~m}^{3}$ of concrete) & 0.375 \\
Water/cement ratio & 0.44 \\
\hline
\end{tabular}

\subsection{Triaxial Test under Moderate Confining Pressure}

3.1.1. Triaxial Behavior of Dry Samples. The triaxial tests performed consist of applying a hydrostatic pressure around the specimen thanks to a noncompressible fluid at a rate of $1.7 \mathrm{MPa} / \mathrm{s}$ (for quasi-static testing) up to a pressure value $p_{\text {conf }}$. This part of the test is called the hydrostatic phase.

After this first hydrostatic phase, a constant displacement rate of $14 \mu \mathrm{m} / \mathrm{s}$ is imposed by the axial jack. During this second part of the test, called the deviatoric phase, the confining pressure $p_{\text {conf }}$ on the lateral face is kept constant. The circumferential strain of the sample is measured using two circumferential gauges. The axial strain is obtained by means of both an axial gauge and an LVDT sensor (to measure the displacement of the upper anvil relative to the lower anvil), providing, respectively, a local measurement and a mean measurement of the strain.

Figure 2 shows the evolution in axial stress as a function of strain for a confining pressure up to $100 \mathrm{MPa}$. In these tests, the degree of concrete saturation equals roughly $60 \%$. For the tests presented herein, the strain measurements recorded by the axial gauge and LVDT sensor remain relatively close during the hydrostatic part, indicating that the sample deforms homogeneously. To improve the clarity of Figure 2, only the axial strains measured by LVDT and the average circumferential strain measurement from the two gauges are presented.

Figure 2 also reveals the increase in concrete stiffness and strength with confining pressure. This phenomenon could be explained by the irreversible closure of porosity (compaction) when the mean stress increases. It is worth noting in these tests that for a confining pressure of less than $47 \mathrm{MPa}$, a stress peak is observed in the axial behavior, whereas this same phenomenon is not observed when testing with a confining pressure of $100 \mathrm{MPa}[4,5,7,14,15]$. At this level of confinement, the concrete behavior becomes ductile and the material failure criterion could not be determined by considering just the axial behavior.

Nevertheless, as proposed by $\mathrm{Vu}$ et al. [6], the limit state could be determined from volumetric measurements during the triaxial tests (Figure 3). A modification indeed 


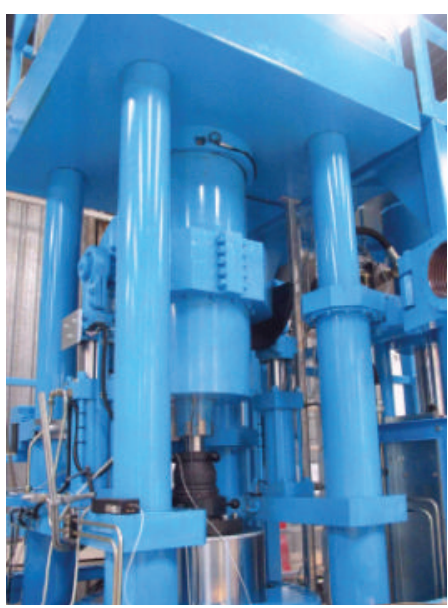

(a)

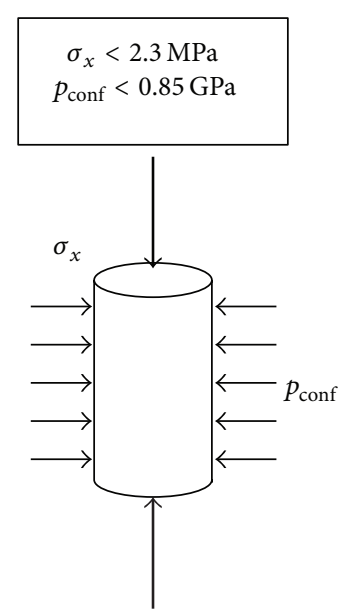

(b)

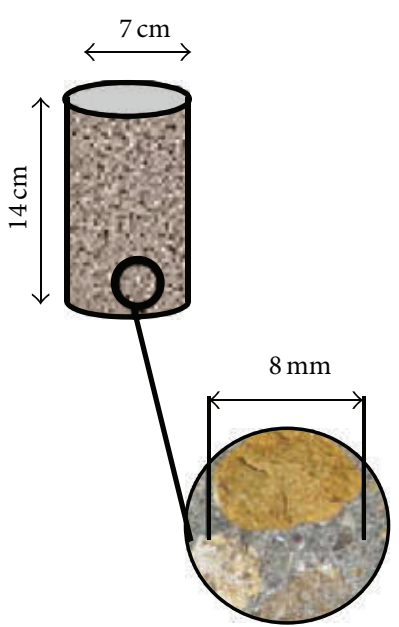

(c)

FIGURE 1: General view of the GIGA press (a), loading capacity (b), and sample sizes (c).

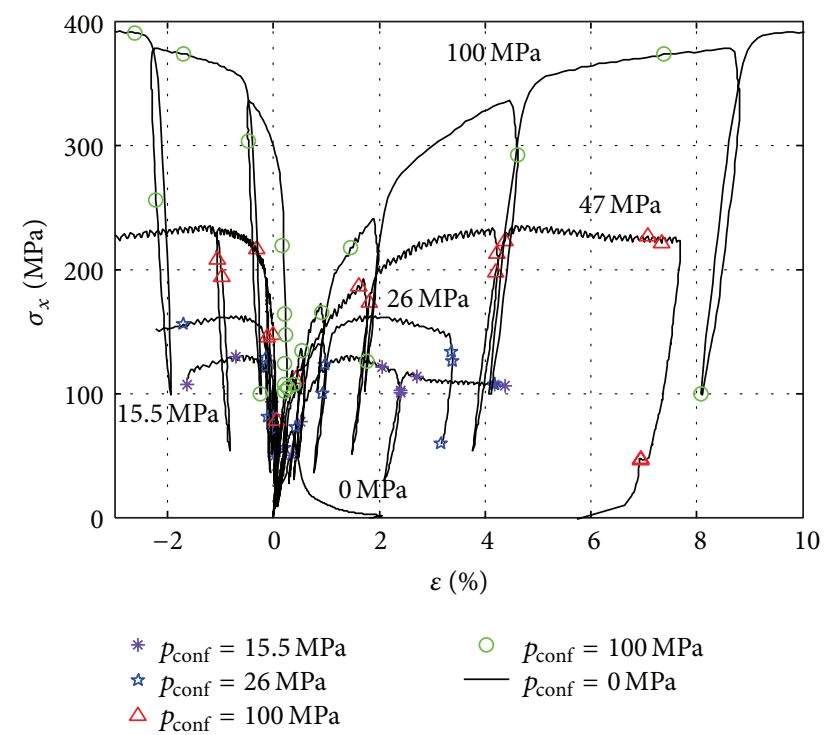

Figure 2: Axial stress versus axial and circumferential strains for various confining pressures (LVDT for axial strains and strain gauges for circumferential strains).

occurs in the volumetric behavior, which reaches a limit state corresponding to the transition from contraction to dilatancy with no softening. On a macroscopic scale, the presence of pores in the sample may suggest shear bands outside the area measured by the axial gauge. For this reason, the volumetric strains must be calculated with the LVDT measurement (total axial strain).

Even though the good level of test repeatability can be visualized during the hydrostatic part (Figures 2 and 3) and has been demonstrated on triaxial tests at very high confining pressure by [6], ensuring the reliability of this analysis requires a validation. Therefore, tests on two samples with a confining pressure of $26 \mathrm{MPa}$ were performed. This confining pressure is in fact quite low in comparison with

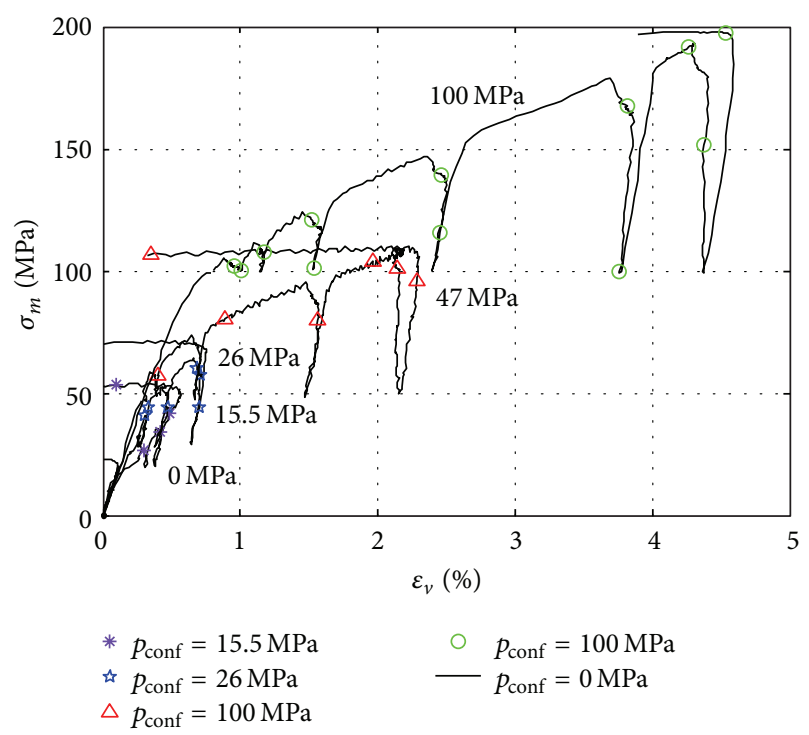

FIGURE 3: Mean stress versus volumetric strain for various confining pressures.

the GIGA press capacity. These results, which describe both the axial behavior and volumetric behavior, are displayed in Figures 4 and 5. The responses of the two samples tested at a $26 \mathrm{MPa}$ confining pressure are close except for the volumetric measurement, which is higher at the end of one of the tests due to the lateral (local) measurement naturally being altered after damage localization.

3.1.2. Influence of Free Water (at Moderate Confining Pressure). A triaxial test at a $50 \mathrm{MPa}$ confining pressure has been performed on a saturated concrete specimen in order to study the influence of the saturation ratio (SR). The procedure for testing saturated samples is described in [5]. The axial strain is measured solely by the LVDT sensor. Figure 6 shows 


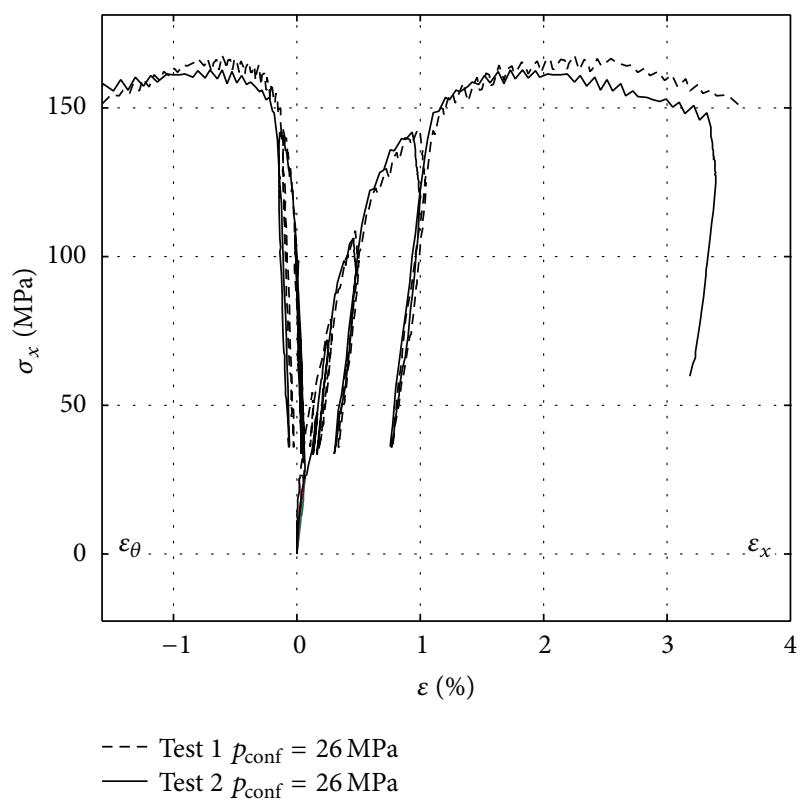

FIgURE 4: Axial stress versus axial and circumferential strains for triaxial tests under $26 \mathrm{MPa}$ of confining pressure (LVDT for axial strains and strain gauges for orthoradial strains).

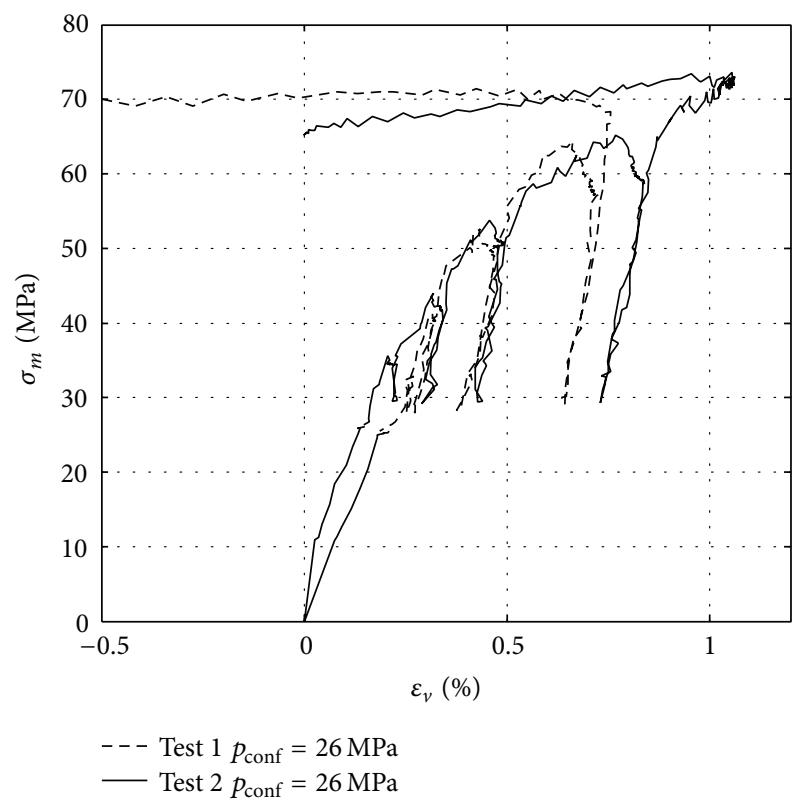

Figure 5: Mean stress versus volumetric strains for triaxial tests under $26 \mathrm{MPa}$ of confining pressure (LVDT for axial strains and strain gauges for orthoradial strains).

a comparison between the evolution of axial stress versus axial strain obtained during this second test ( $\mathrm{SR}=100 \%)$ and the same evolution obtained on wet concrete $(\mathrm{SR}=60 \%)$ at a $50 \mathrm{MPa}$ confining pressure. The maximum axial stress is approximately $240 \mathrm{MPa}$ in both tests (deviatoric stress $=190 \mathrm{MPa}$ ). This result, which agrees with that found on a standard concrete [4], confirms that the saturation ratio

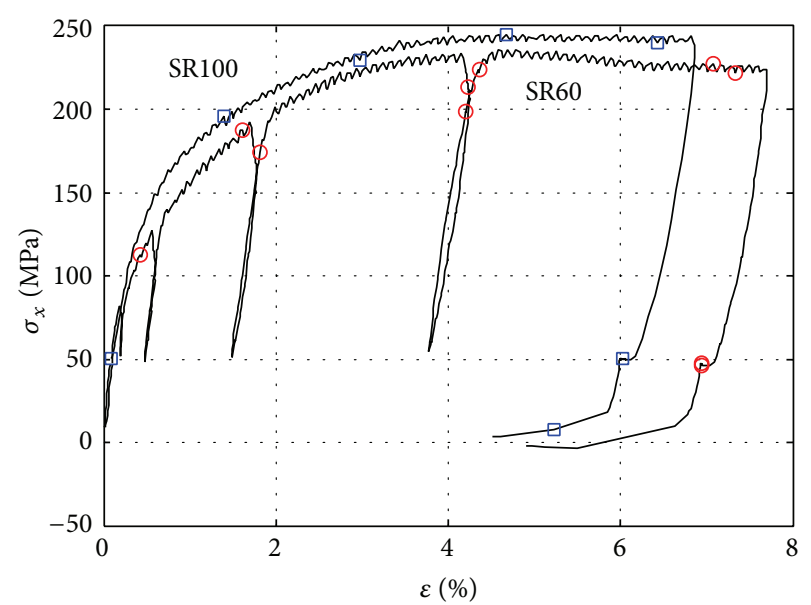

Figure 6: Comparison of axial behavior at $50 \mathrm{MPa}$ confining pressure for two saturation ratios (curve with circles: $\mathrm{SR}=60 \%$, $p=47 \mathrm{MPa}$; curve with squares: $\mathrm{SR}=100 \%, p_{\text {conf }}=50 \mathrm{MPa}$ ).

seems to have little influence on the triaxial behavior of concrete at moderate confining pressures.

\subsection{Triaxial Test under High Confining Pressure}

3.2.1. Influence of Free Water (under High Confining Pressure). The previous section demonstrated that the influence of saturation ratio remains limited at moderate confining pressures. Nevertheless, in case of impact, the concrete may be subjected to very high triaxial stresses [15-17], and additional triaxial tests are becoming necessary to investigate the influence of saturation ratio on the triaxial concrete response. Figure 7 compares two hydrostatic tests at a high confining pressure with two different saturation ratios (60\% and $100 \%)$.

The concrete responses displayed in Figure 7 are very close for mean stress levels less than $100 \mathrm{MPa}$; this zone corresponds to the elastic behavior of VTT concrete. Beyond this zone, the concrete porosity begins to close and the volumetric strains obtained for a saturated concrete (i.e., degree of saturation near $100 \%$ ) increase less than those of concrete with a $60 \%$ saturation ratio. This phenomenon is explained by the infilling of pores with water for the saturated concrete sample and by the fact that the bulk modulus of water is higher than the one of air. Thus, the volumetric strain of saturated concrete is lower than that of dried concrete for the same mean stress value.

In the event of an impact load, the concrete is not only subjected to a hydrostatic loading but also subjected to shear stresses. Figure 8 compares the stress deviator with respect to axial strains during the deviatoric phase. This figure highlights another important influence of free water content under high confining pressure. The water content increase induces a decrease in axial strength. At the same confining pressure, this also corresponds to a decrease in shear strength as a result of the lubrication effect of water. When all pores are closed under the effect of compaction due to triaxial loading, the cement matrix with the presence of water in the pores infilled by water is less resistant to shear compared 


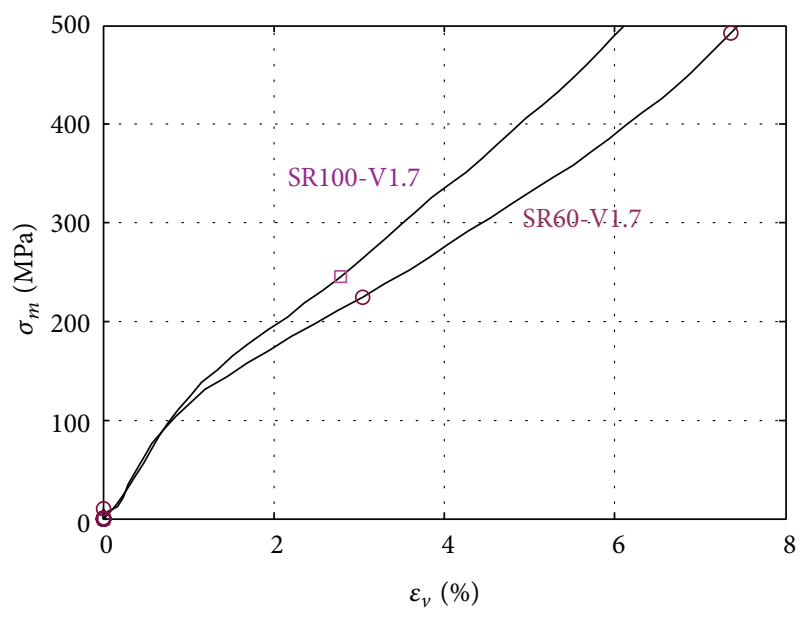

Figure 7: Hydrostatic behavior of VTT concrete: effect of the saturation ratio $(\mathrm{SR})$ (curve with circles: $\mathrm{SR}=60 \%$; curve with squares: $\mathrm{SR}=100 \%)$.

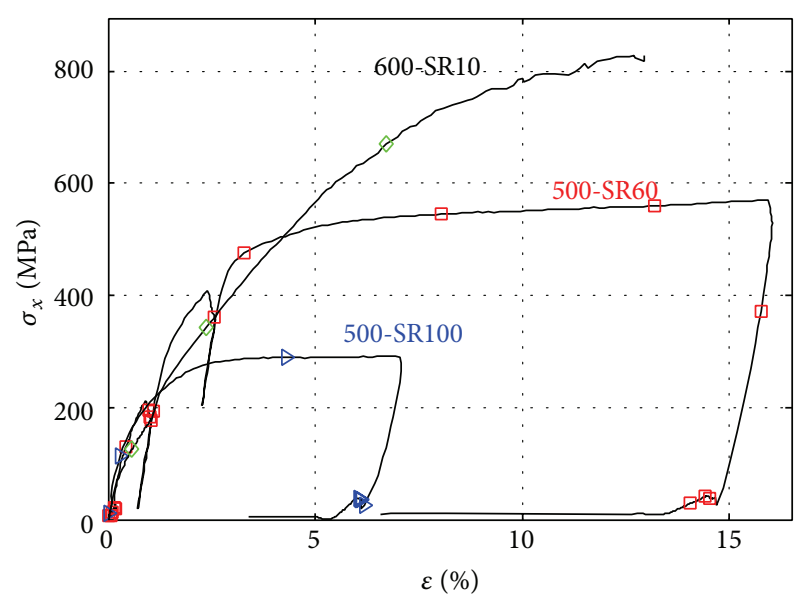

FIGURE 8: Axial behavior, comparison of the shear behavior of concrete for several saturation ratios (curve with circles: $\mathrm{SR}=10 \%$; curve with squares: $\mathrm{SR}=60 \%$; curve with triangles: $\mathrm{SR}=100 \%$ ).

to the compacted cementitious matrix. This phenomenon was also observed by $\mathrm{Vu}$ et al. [5]. For moderate confining pressures, the compaction is not sufficient to provoke an interstitial fluid pressure effect and no influence of the free water on the concrete behavior is observed.

3.2.2. Effect of Loading Rate. Two tests with different rates were performed on concrete cured under the same conditions (i.e., an SR equal to about 60\%). For the test with the faster loading rate $(1.7 \mathrm{MPa} / \mathrm{s})$, the mean stress is higher than the one with the lowest loading rate $(0.5 \mathrm{MPa} / \mathrm{s})$ (Figure 9). The difference between the two curves might be due to concrete creep, which tends to increase deformation during the slower test. Creep strains can be visualized on the cyclic test when the mean stress is kept constant (at a mean stress of $400 \mathrm{MPa}$ ). Knowing that the volume of cement paste is close to $37.5 \%$ of the concrete volume and that the concrete is wet, significant

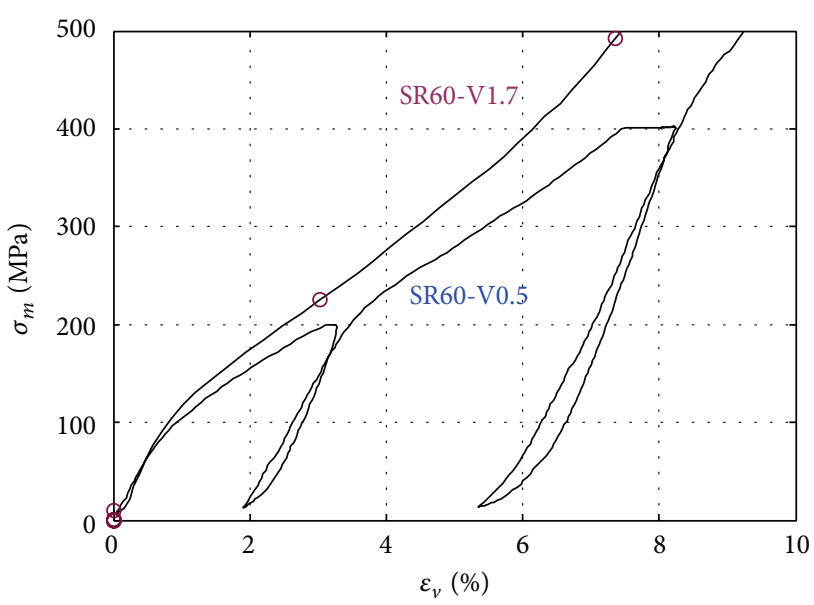

FIGURE 9: Hydrostatic behavior of VTT concrete at two loading rates $(\mathrm{V} 1.7=1.7 \mathrm{MPa} / \mathrm{s}, \mathrm{V} 0.5=0.5 \mathrm{MPa} / \mathrm{s})$.

creep strains are expected [18-20], and these should be subtracted from the results before identifying the concrete behavior for dynamic loadings.

3.2.3. Concrete Shear Limit State. The shear limit state of concrete subjected to triaxial loading depends on both the confinement level and the free water content. For moderate confining pressures, the water content has little influence on the limit state. The shear limit state is defined as the maximum deviatoric stress obtained under triaxial loading. This maximum stress however is not observed at high confining pressures, especially for dry samples. As briefly explained above, another criterion should be used: Vu et al. [5] proposed determining this limit state on the basis of volumetric strain. Regardless of the level of containment, once a maximum volumetric strain of contraction has been reached, expansion occurs. The material limit state is then defined as the maximum volumetric strain reached during a test. This point is called the transition point between contraction and dilatancy, and the deviatoric stress obtained at the maximum volumetric strain corresponds to the shear stress limit state of concrete. This value strongly depends on the level of confinement applied as well as the degree of saturation of the concrete sample [2]. At a moderate confining pressure, this transition also corresponds to the peak stress.

The measured limit states are plotted on Figures 10 and 11 in the deviatoric stress/mean stress plane for all tests previously described (from $0 \mathrm{MPa}$ to $600 \mathrm{MPa}$ and for various degrees of saturation). Figure 11 offers a close-up of Figure 10 at low confining pressures.

Figure 10 reveals that, for a given mean stress, the maximum deviatoric stress reached during the test strongly depends on the saturation ratio of the concrete specimen. The presence of free water limits the admissible shear stress of concrete in the presence of confining pressure.

For a moderate confining pressure (i.e., less than $50 \mathrm{MPa}$ ), the influence of free water on concrete behavior seems to be limited, whereas the influence of the degree of saturation is significant at a confining pressure on the order of 


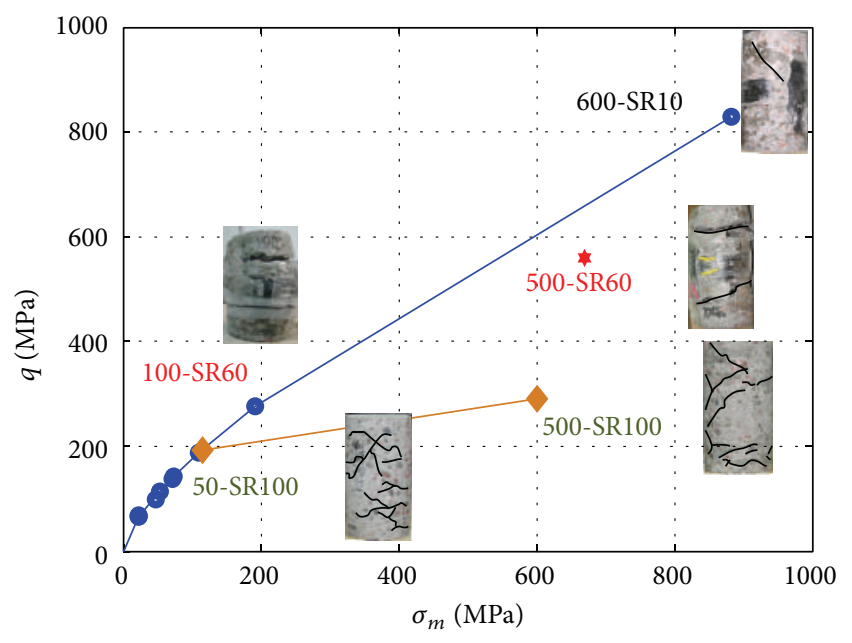

FIGURE 10: Shear limit state of concrete and failure patterns of samples: maximum deviatoric stress $(q)$ versus mean stress for various tests (c: cyclic loading).

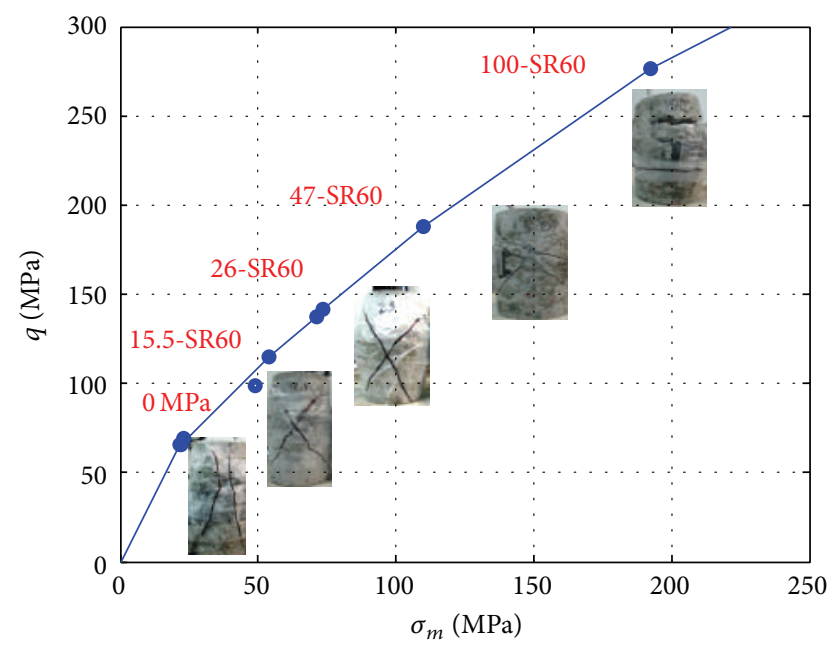

FIGURE 11: Close-up of Figure 10 for confining pressures of less than $100 \mathrm{MPa}$.

$500 \mathrm{MPa}$. Consequently, the concrete shear stress limit is highly dependent not only on confining pressure but also on the concrete free water content as well. This important result may exert a major effect on the response of a concrete structure subjected to an impact and should be taken into account when modeling concrete behavior.

The failure patterns of concrete samples are also presented in Figures 10 and 11. Cracks are oriented in the vertical direction during unconfined compression. As the confining pressure increases, these cracks rotate into the horizontal direction. The horizontal rupture pattern is known as compaction bands.

The presence of free water seems to also have an impact on the failure pattern. Whereas for specimens with $60 \%$ saturation ratio the failure pattern could be assimilated to a compaction band (more or less clearly depending on the reached dilatancy rate), the failure pattern of saturated concrete is, on the other hand, clearly composed of a macroscopic crack network. This modification is probably due to the presence of water in the cement matrix, which as a result could not be compacted.

\section{Modeling the Triaxial Behavior of Concrete}

4.1. General Description of the PRM Coupled Model. The PRM coupled model was developed by Pontiroli et al. [10] in order to deal with computational problems of structures subjected to impact or blast loading. This model is based on a coupling between an elastic-damageable model [21] and a plasticity model initially developed for soils [22]; it includes a calculation of the effective stress defined in [23] for wet concrete, in order to take into account the influence of water saturation on the response of concrete. The damage model is based on two damage variables in compression and in tension, respectively, that simulate the unilateral feature of concrete behavior at low confining pressure. The plasticity model can correctly reproduce the mechanism of irreversible pore closure during compaction. The yield limit, defined as deviatoric stress $(q) /$ mean stress $\left(\sigma_{m}\right)$, is assumed to correspond to the limit state of the material discussed in the previous section.

4.2. Model Improvement. The PRM coupled model allows obtaining a good prediction of concrete behavior under impact loading for thin slabs [10] because on one hand only moderate confining pressures are generated under the impact zone and one the other hand thin slabs dry quickly. Nevertheless, several shortcomings exist in the present model [10] leading to underestimating the volumetric strain measurements and a postcompaction linear behavior which is not representative of experimental behavior and would exert a significant impact on the predicted behavior of thick slab or massive structures.

4.2.1. Influence of the Deviatoric Stress on Volumetric Behavior. The plasticity model assumes that inelastic volumetric and shear strains are obtained independently. The volumetric strain $\left(\varepsilon_{v}\right)$ is assumed to depend on just the mean stress $\left(\sigma_{m}\right)$, while the strain deviator tensor is obtained by means of a perfectly plastic damage model.

The effect of the deviatoric stress $q$ on the volumetric behavior of concrete has therefore not been taken into account in the original PRM coupled model (PRM-O). This original model assumes that the compaction curve, that is, the volumetric strain $\left(\varepsilon_{v}\right)$ versus mean stress $\left(\sigma_{m}\right)$ curve, is obtained from material data independently of the loading path. Figure 3 shows that the inelastic volumetric strain depends on both $q$ and $\sigma_{m}$, which suggests the necessity of including the influence of $q$ in the material compaction curve (whereby $\varepsilon_{v}$ is a function of $\left(\sigma_{m}, q\right)$ ).

To improve this PRM model, the curve depicting the volumetric behavior of concrete is not assumed to be bijective; instead, it is assumed to be bounded by both the hydrostatic and oedometric curves (Figure 12). According to test results [2-9], it is indeed assumed that maximum 


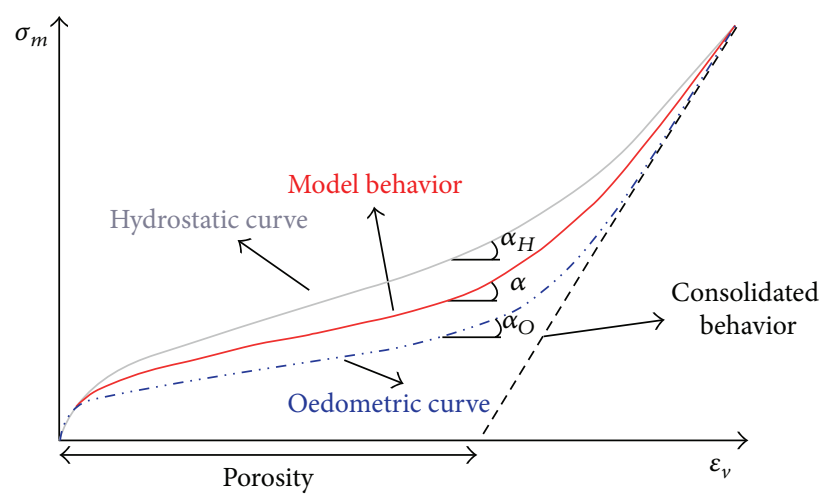

FIGURE 12: Hydrostatic and oedometric constitutive behaviors and resulting triaxial behavior of concrete; mean stress versus volumetric strain.

compaction is obtained under an oedometric loading path. Under uniaxial strain conditions, concrete compaction is maximized because dilatancy is being prevented, whereas the hydrostatic loading path yields a lower compaction. The compaction curve oedometric test and the hydrostatic curve are then to be used as input data due to their ease of access by experimental measurement.

The variation in mean stress $\sigma_{m}$ between the bounded curves is then given by the following:

$$
d \sigma_{m}=\alpha d \varepsilon_{v}
$$

with

$$
\alpha=\alpha_{H}+\left(\alpha_{o}-\alpha_{H}\right) \operatorname{Min}\left[\left(\frac{\left(d q / d \sigma_{m}\right)}{\left(d q / d \sigma_{m}\right)_{o}}\right) ; 1\right],
$$

where (see Figure 12) $\alpha_{H}=d \sigma_{m} / d \varepsilon_{v}$ obtained from a hydrostatic test; $\alpha_{o}=\left(d \sigma_{m} / d \varepsilon_{v}\right)_{o}$ obtained from an oedometric test; $d q / d \sigma_{m}=$ load path direction at the current Gauss point; $\left(d q / d \sigma_{m}\right)_{o}=$ oedometric load path direction obtained from an oedometric test.

In formulae (1) and (2), the volumetric strain $\varepsilon_{v}$ depends on both the mean stress $\sigma_{m}$ and deviatoric shear stress $q$. A hydrostatic loading path contains no shear stress and its behavior follows the hydrostatic curve; however, for a current triaxial loading path with shear stress, the level of compaction is increased.

\subsubsection{Influence of the Water Saturation Ratio on Volumetric} Behavior. Two types of approaches are available to characterize the behavior of a porous medium at its homogenized scale from microscopic-level properties. Firstly, the "mixing law" approach takes into account, at the microscopic level, the interaction between the two phases (liquid + solid) by means of simple rheological models for each phase, whether they are associated in series or associated in parallel . Secondly, the poromechanical approach [24] assumes that the mechanics concepts in continuum mechanics are still valid at the macroscopic scale when the two phases (liquid + solid) overlap.
In the original PRM coupled model, the concept of effective stress is applied to take into account the presence of water in confined concrete when using the first approach. The drawback with such an approach is that the material behavior becomes elastic after reaching the consolidation point (once all open pores are closed), which is not observed experimentally. In the improved model, the poromechanical approach allows taking the effect of free water into account.

The studied porous medium is assumed to be composed of both a solid phase (skeleton) and a fluid phase occupying the voids [24]. The concept of effective stress is introduced to separate fluid pressure in the total pressure calculation:

$$
\sigma_{\text {tot }}=\sigma_{M}+b p
$$

with $\sigma_{\text {tot }}$ being the total stress, $\sigma_{M}$ being transmitted by the matrix at a macroscopic scale, $p$ being the pore pressure, and $b$ being the Biot coefficient, which depends on the nature of the porosity.

The calculation of pore pressure $p$ is based on the MieGrüneisen equation of state, which is

$$
p=\frac{\rho_{0} C_{0}^{2}\left(\varepsilon_{v}-\varepsilon_{v p s}\right)}{\left(1-s\left(\varepsilon_{v}-\varepsilon_{v p s}\right)\right)^{2}}\left[1-\frac{\Gamma_{0}\left(\varepsilon_{v}-\varepsilon_{v p s}\right)}{2}\right]+\Gamma_{0} \rho_{0} E_{M},
$$

where $C_{0}$ is the speed of sound $\left(C_{0}=1,500 \mathrm{~m} / \mathrm{s}\right), \varphi_{0}$ is the density ( $\varphi_{0}=1,000 \mathrm{~kg} / \mathrm{m}^{3}$ for water), $s$ and $\Gamma_{0}$ are two Mie-Grüneisen coefficients $\left(s=1.75\right.$ and $\Gamma_{0}=0.28$ for water), and $E_{M}$ is the internal energy per unit mass, with this energy being considered negligible for water temperature and ambient pressure.

$\sigma_{M}$ and $b$ can be obtained by applying the following formulae [24]:

$$
\begin{aligned}
& \sigma_{M}=K_{0} \varepsilon_{v}, \\
& b=1-\frac{K_{0}}{K_{s}},
\end{aligned}
$$

where $K_{0}$ is the modulus of the drained material, $\varepsilon_{v}$ is the volumetric strain at the homogenized scale, and $K_{s}$ is the compressibility modulus of the skeleton.

From (6), in the particular case where $K_{0} \ll K_{s}, b$ is then close to 1 , a result that simplifies (3) and becomes $\sigma_{\text {tot }}=$ $\sigma_{M}+p$ (i.e., Terzaghi formula). In contrast, when $K_{0} \approx K_{s}$ (case of dry concrete), $b$ tends to 0 . Thanks to homogenization of the drained porous medium [24], the ratio $K_{0} / K_{s}$ can be estimated as follows:

$$
\frac{K_{0}}{K_{s}}=(1-\phi)^{3},
$$

where $\phi$ is the porosity of the porous medium at the current state.

With this new hypothesis, whenever the material reaches the point of consolidation (i.e., void pores become closed), the volumetric behavior remains nonlinear due to the fact that the voids filled with water continue to be compressed under compaction. Another advantage of this model improvement is the unique point of consolidation instead of two points in the original PRM model (Figure 13). 


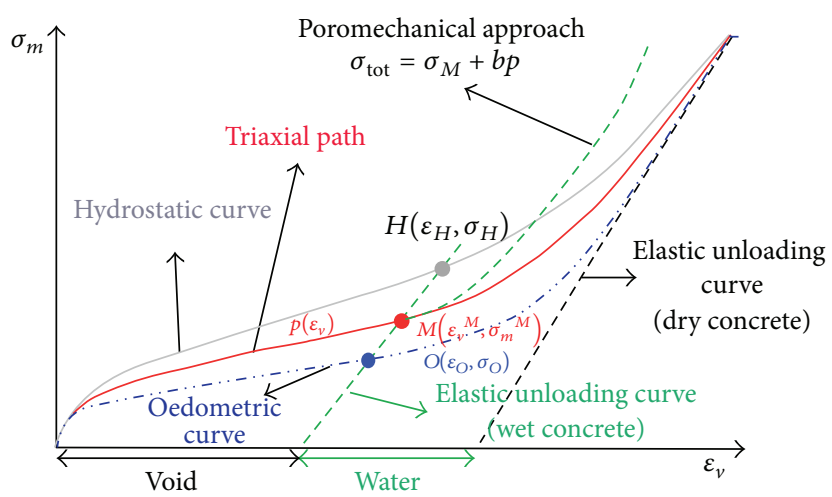

FIGURE 13: Stress calculation diagram according to the poromechanical approach, as the concrete consolidates.

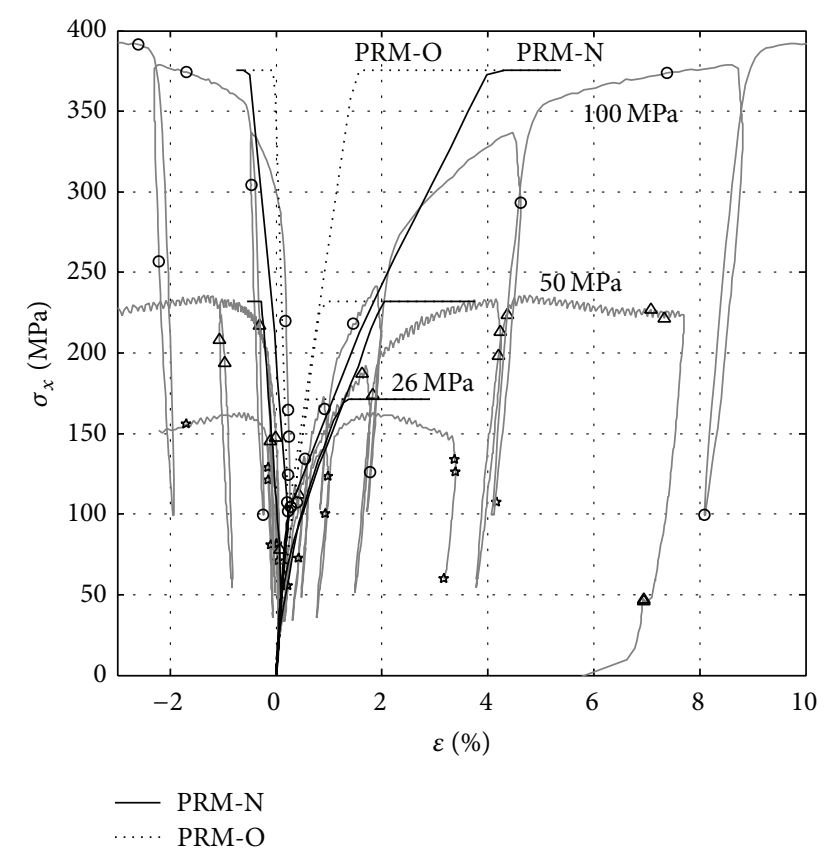

FIGURE 14: Axial stress versus axial and circumferential strains: comparisons of experimental findings with simulation results obtained from the original (PRM-O) and new (PRM-N) models for a wet concrete specimen under moderate confining pressure.

4.3. Comparison between Experimental Results and Test Simulations. The simulation results obtained with the original PRM coupled model as well as with the new model are compared to experimental results in Figures 14 through 16.

4.3.1. Wet Concrete. Figures 14 and 15 show results for a concrete specimen with a $60 \%$ saturation degree subjected to triaxial compression with confining pressures varying from 15 to $100 \mathrm{MPa}$. For this saturation ratio and due to the moderate confining pressure, the concrete behavior is not at all affected by the free water. The initial PRM coupled model allows for a good prediction of the maximum stress; however, the strains have been significantly underestimated. By taking into account the influence of the deviatoric stress on the

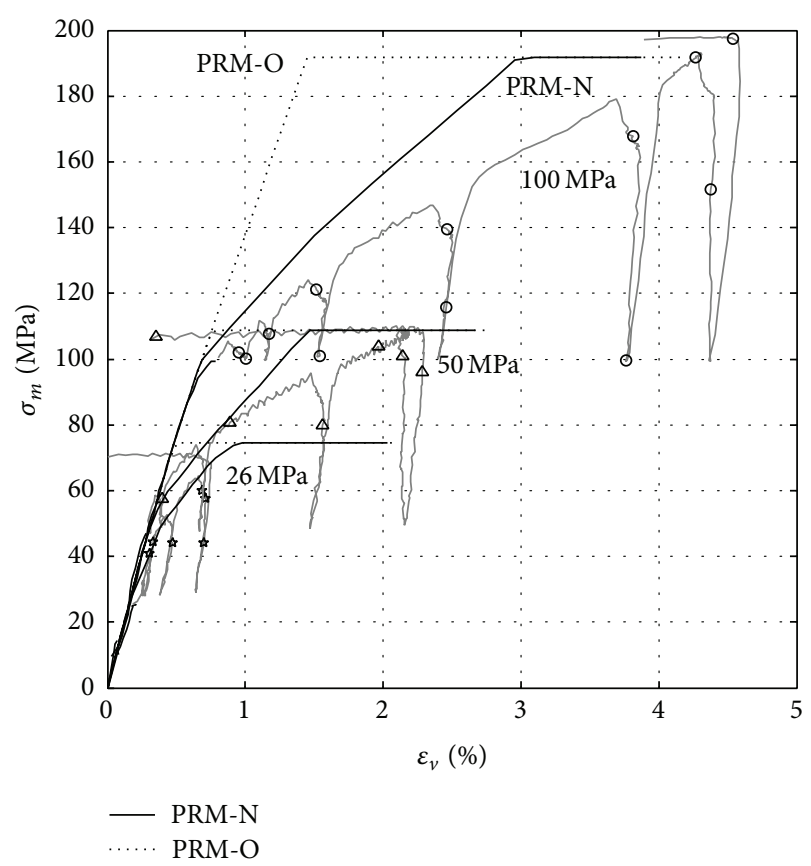

FIGURE 15: Mean stress versus volumetric strain: comparisons of experimental findings with simulation results obtained from the original (PRM-O) and new (PRM-N) models for a wet concrete specimen under moderate confining pressure.

volumetric behavior of concrete the prediction of both the axial and volumetric strains greatly improves.

4.3.2. Saturated Concrete. Figure 16 shows the experimental volumetric behavior results of saturated concrete and dry concrete, along with their comparisons to simulation results output from both the original and new PRM coupled models. The original model considers an elastic postconsolidation behavior (closure of voids), while the modified model provides simulation results closer to the experimental findings.

\section{Conclusion}

This paper has presented new experimental results performed on a high performance concrete specimen tested within the framework of the IRIS project, along with the simulation of these tests using the PRM coupled model improved to fit the experimental results better.

Triaxial compression tests were performed at both moderate and high confining pressures on concrete specimens with two saturation ratios. Significant differences in the maximum stresses attained have been highlighted. For a moderate confining pressure (i.e., less than $50 \mathrm{MPa}$ ), the influence of free water on concrete behavior indeed seems to be quite small, whereas the influence of the degree of saturation is significant at a high confining pressure $(500 \mathrm{MPa})$. The volumetric strains are lower under hydrostatic loading for saturated concrete, though the water also tends to limit the shear stress level. Moreover, the water modifies failure patterns for the saturated samples. 


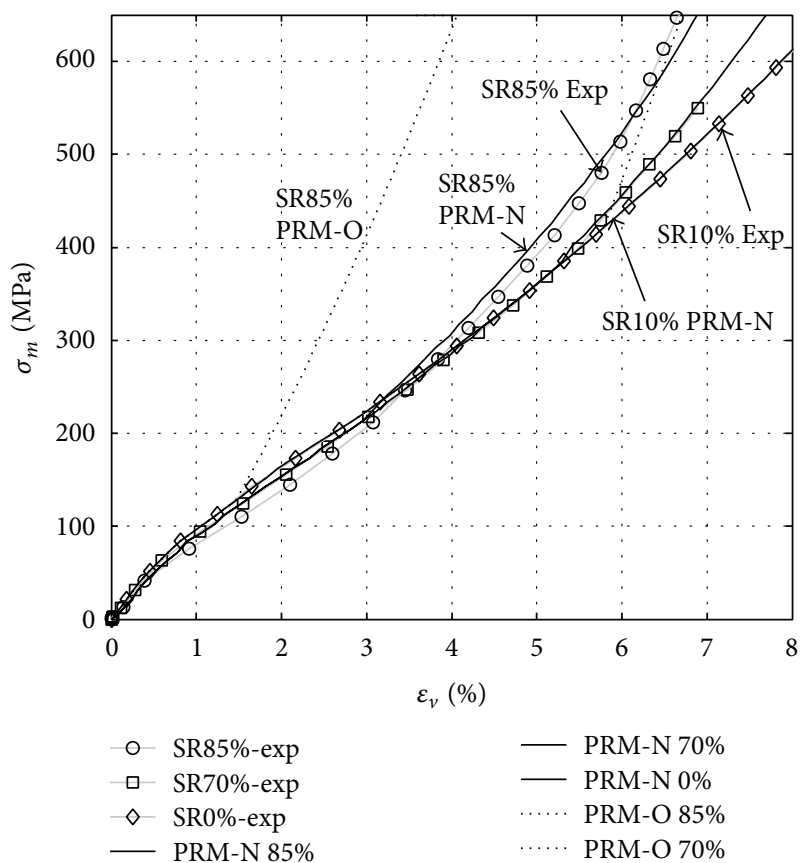

FIGURE 16: Mean stress versus volumetric strain: comparisons of experimental findings with simulation results obtained from the original (PRM-O) and new (PRM-N) models for saturated concrete and dry concrete under high confining pressure.

This paper has also provided some limitations associated with the PRM model, and a number of improvements have been proposed. The modified PRM model takes into account the influence of deviatoric stress on volumetric behavior. The influence of the saturation ratio on concrete behavior under triaxial compression has been modified as well thanks to the poromechanical approach that yields a unique consolidation point and a more realistic concrete behavior for wet concrete beyond this point. These changes have considerably improved the concrete behavior prediction under triaxial compression. Such improvements may exert a major effect on the response of a concrete structure subjected to an impact.

\section{Conflict of Interests}

The authors declare that there is no conflict of interests regarding the publication of this paper.

\section{Acknowledgments}

This research project was supported by both the French Institute of Radio-Protection and Nuclear Safety (Institut de Radioprotection et de Sureté Nucléaire, IRSN) and by CEA Gramat. The authors would like to thank Dr. Eric Buzaud and Dr. C. Pontiroli of the CEA-Gramat Center for the very helpful scientific exchanges throughout this study.

\section{References}

[1] V. Baroghel-Bouny, M. Mainguy, T. Lassabatere, and O. Coussy, "Characterization and identification of equilibrium and transfer moisture properties for ordinary and high-performance cementitious materials," Cement and Concrete Research, vol. 29, no. 8, pp. 1225-1238, 1999.

[2] X. H. Vu, Y. Malecot, L. Daudeville, and E. Buzaud, "Experimental analysis of concrete behavior under high confinement: effect of the saturation ratio," International Journal of Solids and Structures, vol. 46, no. 5, pp. 1105-1120, 2009.

[3] E. Piotrowska, Y. Malecot, and Y. Ke, "Experimental investigation of the effect of coarse aggregate shape and composition on concrete triaxial behavior," Mechanics of Materials, vol. 79, pp. 45-57, 2014.

[4] T. Gabet, Y. Malécot, and L. Daudeville, "Triaxial behaviour of concrete under high stresses: influence of the loading path on compaction and limit states," Cement and Concrete Research, vol. 38, no. 3, pp. 403-412, 2008.

[5] X. H. Vu, Y. Malecot, and L. Daudeville, "Strain measurements on porous concrete samples for triaxial compression and extension tests under very high confinement," The Journal of Strain Analysis for Engineering Design, vol. 44, no. 8, pp. 633-657, 2008.

[6] X. H. Vu, Y. Malecot, L. Daudeville, and E. Buzaud, "Effect of the water/cement ratio on concrete behavior under extreme loading," International Journal for Numerical and Analytical Methods in Geomechanics, vol. 33, no. 17, pp. 1867-1888, 2009.

[7] C. Poinard, Y. Malecot, and L. Daudeville, "Damage of concrete in a very high stress state: experimental investigation," Materials and Structures, vol. 43, no. 1-2, pp. 15-29, 2010.

[8] Y. Malecot, L. Daudeville, F. Dupray, C. Poinard, and E. Buzaud, "Strength and damage of concrete under high triaxial loading," European Journal of Environmental and Civil Engineering, vol. 14, no. 6-7, pp. 777-803, 2010.

[9] X. H. Vu, L. Daudeville, and Y. Malecot, "Effect of coarse aggregate size and cement paste volume on concrete behavior under high triaxial compression loading," Construction and Building Materials, vol. 25, no. 10, pp. 3941-3949, 2011.

[10] C. Pontiroli, A. Rouquand, and J. Mazars, "Predicting concrete behaviour from quasi-static loading to hypervelocity impact. An overview of the PRM model," European Journal of Environmental and Civil Engineering, vol. 14, no. 6-7, pp. 703-727, 2010.

[11] http://www.oecd-nea.org/nsd/docs/2011/csni-r2011-8.pdf.

[12] L. Daudeville and Y. Malécot, "Concrete structures under impact," European Journal of Environmental and Civil Engineering, vol. 15, no. 1, pp. 101-140, 2011.

[13] H. Zhao and G. Gary, "On the use of SHPB techniques to determine the dynamic behavior of materials in the range of small strains," International Journal of Solids and Structures, vol. 33, no. 23, pp. 3363-3375, 1996.

[14] E. M. Williams, S. A. Akers, and P. A. Reed, "Laboratory characterization of SAM-35 concrete," Tech. Rep. ERDC/GSL TR-06-150, US Army Engineer Research and Development Center, 2006.

[15] T. L. Warren, A. F. Fossum, and D. J. Frew, "Penetration into low-strength (23 MPa) concrete: target characterization and simulations," International Journal of Impact Engineering, vol. 30, no. 5, pp. 477-503, 2004.

[16] T. Sugano, H. Tsubota, Y. Kasai et al., "Full-scale aircraft impact test for evaluation of impact force," Nuclear Engineering and Design, vol. 140, no. 3, pp. 373-385, 1993.

[17] J. K. Gran and D. J. Frew, "In-target radial stress measurements from penetration experiments into concrete by ogive-nose steel projectiles," International Journal of Impact Engineering, vol. 19, no. 8, pp. 715-726, 1997. 
[18] J. Brooks, Advance Concrete Technology: Concrete Properties, section 7, 2003.

[19] A. M. Neville, "Creep of concrete as a function of its cement paste content," Magazine of Concrete Research, vol. 16, no. 46, pp. 21-30, 1964.

[20] P. Acker and F.-J. Ulm, "Creep and shrinkage of concrete: physical origins and practical measurements," Nuclear Engineering and Design, vol. 203, no. 2-3, pp. 143-158, 2001.

[21] J. F. Dubé, G. Pijaudier-Cabot, and C. La Borderie, "Rate dependent damage model for concrete in dynamics," Journal of Engineering Mechanics, vol. 122, no. 10, pp. 939-947, 1996.

[22] R. D. Krieg, "A simple constitutive description for soils and crushable foams," Tech. Rep. SC-DR-72-0883, Sandia National Laboratories, Albuquerque, NM, USA, 1972.

[23] C. Mariotti, J. P. Perlat, and J. M. Guérin, "A numerical approach for partially saturated geomaterials under shock," International Journal of Impact Engineering, vol. 28, no. 7, pp. 717-741, 2003.

[24] O. Coussy, Mechanics of Porous Continua, John Wiley \& Sons, New York, NY, USA, 1995. 


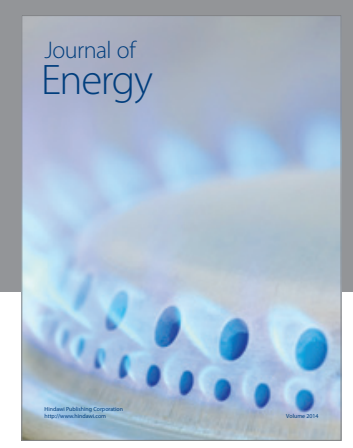

Journal of

Industrial Engineering
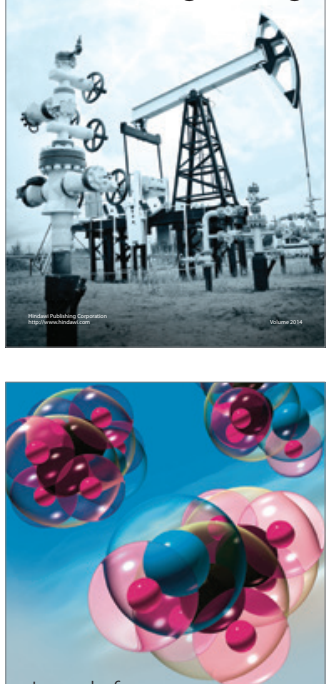

Fuels
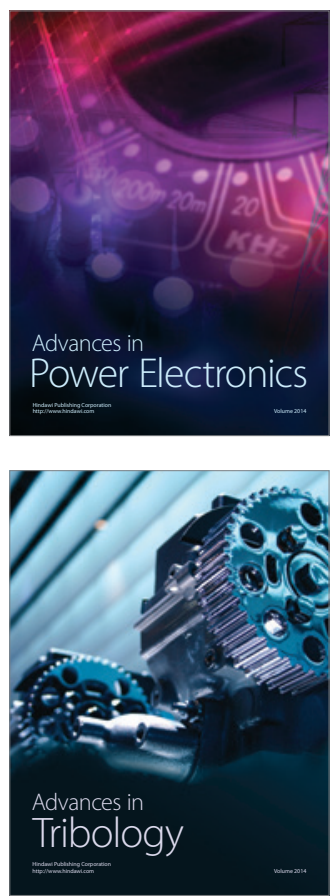

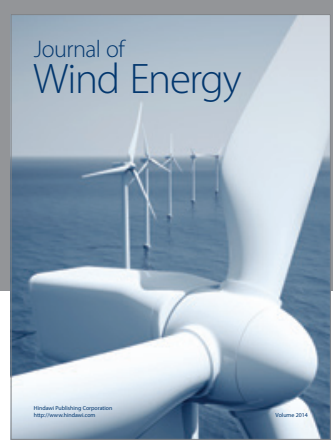

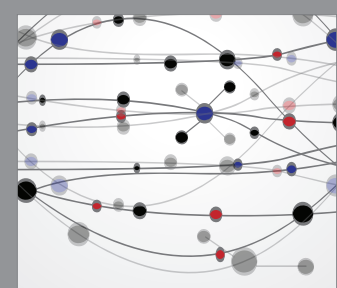

The Scientific World Journal

Submit your manuscripts at http://www.hindawi.com

Journal of

Structures
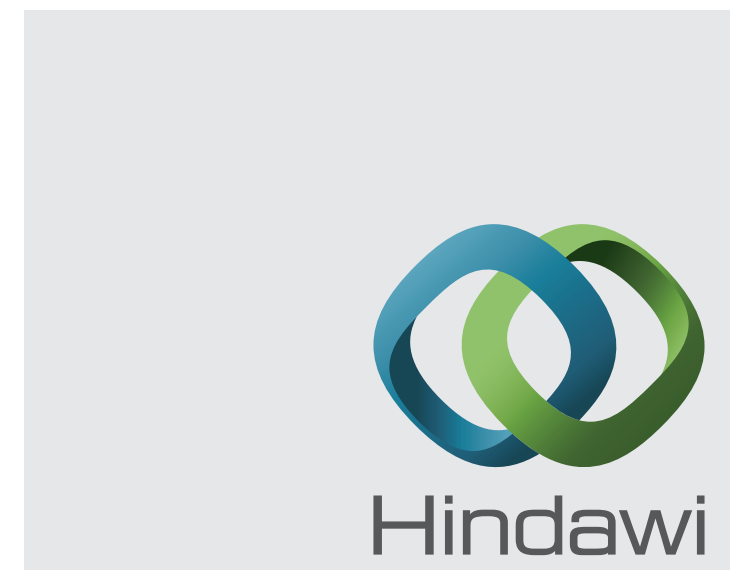

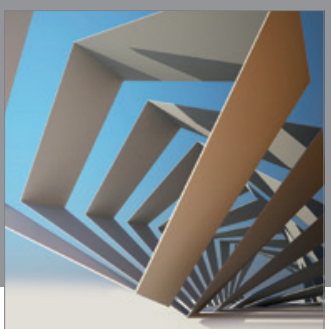

Rotating

Machinery
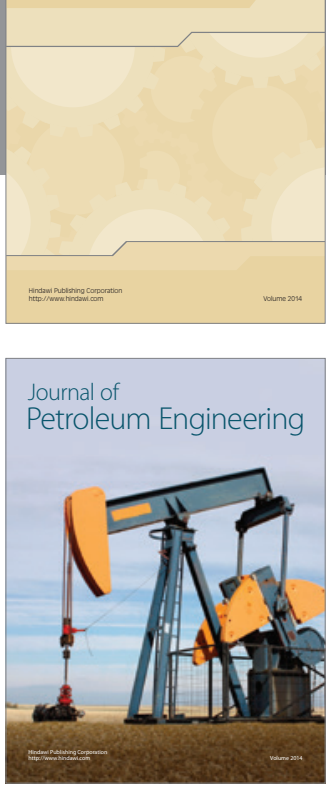

Journal of

Solar Energy
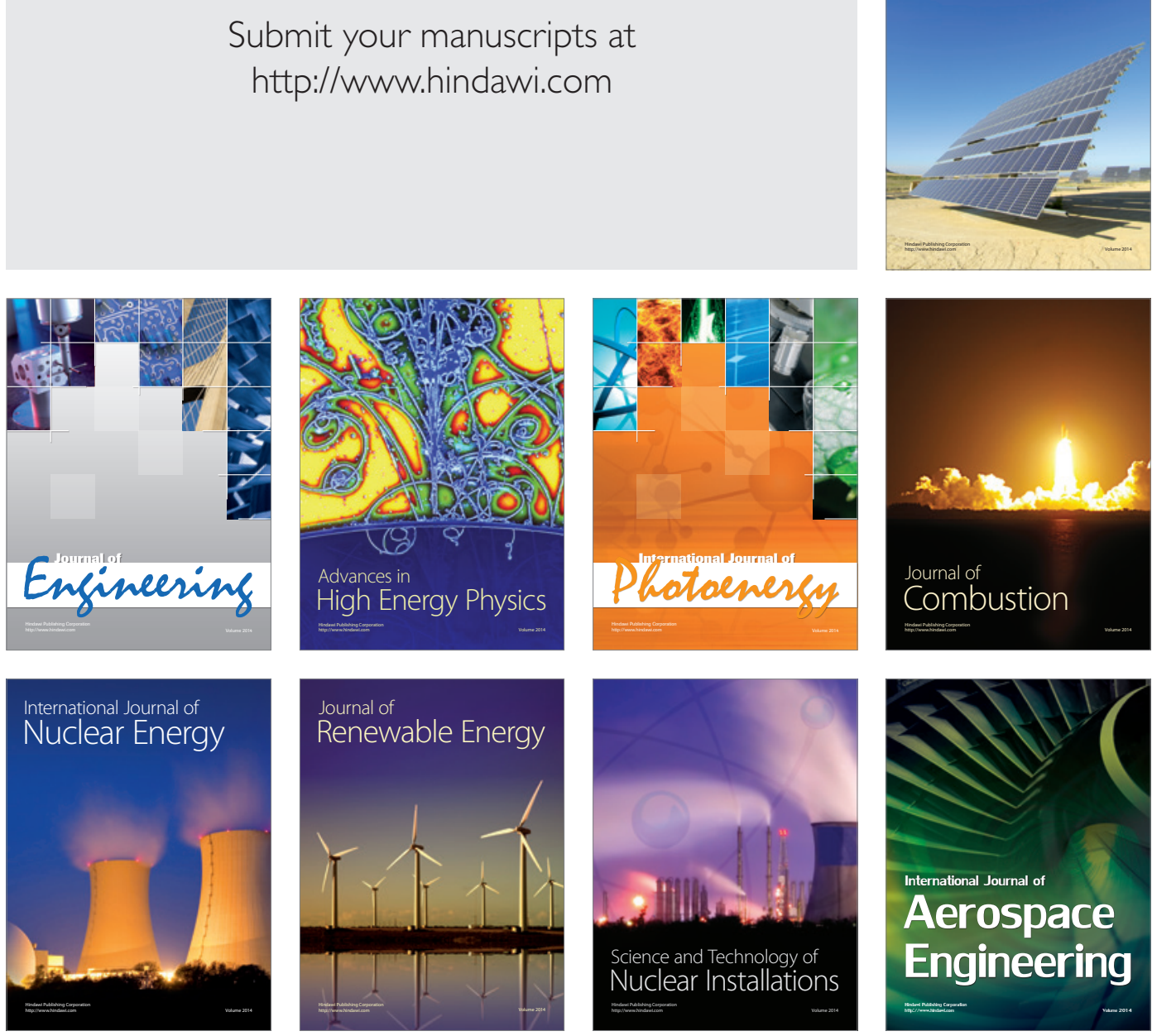